\title{
Una nueva especie de Gnamptogenys (Hymenoptera: Formicidae) y comentarios sobre las especies del género en Colombia y Ecuador
}

\author{
John E. Lattke ${ }^{1}$, Fernando Fernandéz C. ${ }^{2} \&$ Edgard E. Palacio ${ }^{3}$ \\ 1. Instituto de Zoología Agrícola, Universidad Central de Venezuela, Apartado 4579, Maracay 2101-A, Venezuela. \\ (piquihuye@fastmail.com) \\ 2. Instituto de Ciencias Naturales, Universidad Nacional de Colombia, Apartado 7495, Bogotá D.C., Colombia. \\ (ffernandezca@unal.edu.co) \\ 3. Fundación Nova Hylaea, Apartado Aéreo 7954, Bogotá D.C., Colombia. (parasitoideus@yahoo.com)
}

\begin{abstract}
A new species of the ant genus Gnamptogenys (Hymenoptera: Formicidae) with comments on the Colombian and Ecuatorian species. The species of the predatory ant genus Gnamptogenys Roger, 1863 from Colombia (42 species) and Ecuador ( 25 species) are diagnosed and presented, including their known geographical distribution. Gnamptogenys enodis, new species from Colombia is described. Gnamptogenys stellae Lattke, 1995 is new record for Colombia.
\end{abstract}

KEYWORDS. Gnamptogenys, Colombia, Ecuador, taxonomy, distribution.

\section{INTRODUCCIÓN}

El género Gnamptogenys Roger, 1863 comprende hormigas cazadoras con amplia distribución en las regiones Neártica, Neotropical, Oriental y Australiana. La primera revisión se debe a BROwN (1958) quien define el género después de una extensa sinonimia y la ubica dentro de la Tribu Ectatommini y además propone una clave para las especies conocidas. BRANDÃo \& LATTKE (1990) y LATTKE (1991) tratan el grupo minuta, y LATTKE (1995) ofrece una nueva revisión para el género en América, aumentando el numero de especies conocidas en el Hemisferio Occidental a 73. Regionalmente LATTKE (1990) estudia las especies de Venezuela y Fernández et al. (1996) ofrecen listados para las especies de Colombia. LATTKE (1994) realiza un análisis cladístico de Ectatommini sensu Brown reubicando los géneros en las tribus Ectatommini s. str., Paraponerini y Proceratiini. LatTKe (2003) revisa las especies del Hemisferio Oriental, reconociendo 50 especies, y propone un cladograma para dichas especies.

Este trabajo presenta por primera vez las especies del género para Colombia y Ecuador, lo cual complementa el estudio de las especies de Venezuela (LATtKe, 1990) y ofrece un panorama relativamente detallado para la fauna del norte de Suramérica.

\section{MATERIAL Y MÉTODOS}

El material para este estudio está depositado en las siguientes colecciones: BMNH, The Natural History Museum, Londres, Inglaterra; CUIC, Cornell University, Ithaca, New York, USA; EPIC, Colección Edgard "El Costeño" Palacio, Bogotá D.C., Colombia; FFIC, Colección Fernando Fernández C., Bogotá D. C., Colombia; IAVH, Instituto de Investigación de Recursos Biológicos Alexander von Humboldt, Villa de Leyva, Colombia; LACM, Los Angeles County Museum of Natural History, Los Angeles, California, USA; MCZC, Museum of Comparative Zoology, Harvard University, Cambridge,
USA; MIZA, Museo del Instituto de Zoología Agrícola, Universidad Central de Venezuela, Maracay, Aragua, Venezuela; MZSP, Museu de Zoologia, Universidade de São Paulo, São Paulo, Brasil; PSWC, Philip S. Ward Collection, University of California, Davis, California, USA; UDVC, Departamento de Biología, Universidad del Valle, Cali, Valle, Colombia; UNCB, Museo de Historia Natural, Instituto de Ciencias Naturales, Universidad Nacional de Colombia, Bogotá D.C., Colombia; USNM, United States National Museum, Washington, D. C., USA; WMIC, Colección W. P. MacKay, University of Texas, El Paso, Texas, USA.

Las siguientes medidas e índices se utilizan en el texto: AC, ancho cefálico, longitud de la cabeza tomada en vista dorsal en una linea media imaginaria, incluyendo el margen anterior de la lámina del clípeo; DO, diámetro ocular, diámetro del ojo compuesto medido en su eje longitudinal en vista lateral cefálica; LC, longitud cefálica, ancho máximo de la cabeza tomado en el mismo plano que AC, sin incluir los ojos; LE, longitud del escapo, longitud del primer segmento antenal, sin incluir el cóndilo basal y la constricción contigua al cóndilo; LM, longitud de la mandíbula; LW, longitud de Weber, longitud diagonal del mesosoma tomada en perspectiva lateral con una linea imaginaria que va desde el margen anterior del pronoto ( in incluir el cuello) hasta la extremidad posterior de la metapleura; IC, índice cefálico, $\mathrm{AC} / \mathrm{LC}$; IE, índice del escapo LE/AC; IM, índice mandibular LM/AC; IO, índice ocular DO/AC. Otras abreviaciones utilizadas: o., obrera; m., macho; Mpio, municipio; P.N.N., Parque Natural Nacional; r., reina; R.F., Reserva Forestal; R.N.N., Reserva Natural Nacional; Vda, Vereda.

\section{Gnamptogenys acuminata Emery, 1896}

Diagnosis. Mandíbulas semi-triangulares; costillas longitudinales sobre el mesosoma y cara propodeal en declive; suturas transversas del dorso mesosomal débiles, visibles solamente con ciertas angulaciones de 
luz; cuerpo café oscuro a negro; coxas y dos tercios basales de los fémures testáceos a café testáceo; tibias, ápices de los fémures, antenas y mandíbulas café.

Material examinado. COLOMBIA, Meta: Villavicencio (Caño El Buque, $480 \mathrm{~m}$ ), 1 o. (MIZA); Nariño: Barbacoas (Tajadas, 1000 m), 1 o., VIII.1994, F. Escobar col. (IAVH).

\section{Gnamptogenys acuta Brown, 1957}

Diagnosis. Similar a G. striatula; 4 a 6 costillas transversas sobre la cara anterior pronotal, resto del mesosoma con costillas longitudinales; costillas longitudinales sobre la cara anterior del pospeciolo; cuerpo café oscuro a negro, patas café.

Material examinado. COLOMBIA, Meta: Mesetas $(780$ m), 1 o. (FFIC); 4 o. (MIZA); Valle: R. F. Escalerete (180 m), 1 o., VIII.1995, B. Usma \& R. Aldana col. (UDVC). ECUADOR, Napo: N Puyo (Pastaza, 935 m), 1 o. (MIZA).

\section{Gnamptogenys alfaroi Emery, 1894}

Diagnosis. Dorso mandibular liso y brillante con algunas puntuaciones; espina metacoxal presente; proceso subpeciolar proyectado anteriormente; segundo tergo gastral con la mitad basal con costillas longitudinales.

Material examinado. COLOMBIA, Cauca: Isla Gorgona (Acueducto), 1 o., IV.1990, M. Baena col. (IAVH). ECUADOR, Guayas: 3 km SO Bucay, 2 o. (MCZC); 2 o. (MIZA)

\section{Gnamptogenys andina Lattke, 1995}

Diagnosis. Similar a G. strigata. Escultura con costillaje aproximado; margen verticeal cóncavo visto de frente; mandíbula triangular, en su mayoría estriada; ojos relativamente pequeños; espiráculo propodeal ligeramente elevado; nodo peciolar posteriormente inclinado; proceso subpeciolar semi-cuadrado, como es en el subgrupo strigata; costillas longitudinales sobre la cara en declive del pospeciolo; costillas transversas débiles sobre el vientre pospeciolar.

Material examinado. COLOMBIA, Caldas: Aguadas (La Playa, 1610 m), 3 o., XI.1995, C. Sarmiento col. (IAVH); 2 o. (MIZA); Caquetá: P.N.N. Picachos $\left(2^{\circ} 48^{\prime} \mathrm{N} 74^{\circ} 51^{\prime} \mathrm{O}, 1560\right.$ m), 1 r, 2 o., 23.IX.1997, F. Escobar \& E. González col. (IAVH); 1 o., 23.IX.1997, F. Escobar \& E. González col. (MIZA); Nariño: Barbacoas (Tajadas, 1000 m), 1 o., 1.VIII.1994, F. Escobar col, $\mathrm{n}^{\circ} 469$ (IAVH); R.N.N. Río Nambí, Inspección Altaquer, Barbacoas, 4 o., J. Hillaire col. (MIZA); Territorio Kofán $\left(00^{\circ} 30^{`} \mathrm{~N} 77^{\circ} 13^{\prime} \mathrm{O}, 1430 \mathrm{~m}\right), 1$ o., 25.IX.1998, E. González col. (IAVH); Valle: Farallones de Cali, El Topacio $(20 \mathrm{~km}$ NO Cali, 1550 m), 23 o., 31.XII.1981, J. Lattke col. (MIZA); Farallones, 2 o., 1.VII.1991, J. Bustos col. (IAVH); P.N.N. Los Farallones, Pico de Aguila, 1 o., 24.V.1992, M. Gamboa col. (MIZA); (vía Cali-Buenaventura, $15 \mathrm{~km} \mathrm{O}$ Cali, $1200 \mathrm{~m}$ ), 18 o., 1.X.1975, J. Lattke col. (MIZA); Pance (cerca Estación CVC, 1700 m), 16 o., 12.XII.1975, J. Lattke col. (MIZA); R.N. Hato Viejo (Inspección Pance, 2250 m), 1 o., J. Hillaire col. (MIZA); Mpio Argelia (Vereda Las Brisas, Finca San Jorge, $1950 \mathrm{~m}$ ), 1 o. 22.I.1983, T. v.d. Hammen col. (IAVH). ECUADOR, Bolívar: 20 km Palzabamba (500-1800 m), 2 o., 18.IX.1987, N. Zavala col. (MCZC, MZSP).

\section{Gnamptogenys annulata Mayr, 1887}

Diagnosis. Cuerpo finamente estriado; estrías sobre la cabeza, promesonoto y segmentos gástricos 2 y 3 longitudinales; dorso del peciolo con estrías arqueadas transversas; mandíbulas semi-triangulares; espina metacoxal ausente; cuerpo café rojizo; coxas amarillo ferruginosas; tibias y fémures amarillos a testáceos aunque apicalmente amarillo ferruginosos a ferruginosos.

Material examinado. COLOMBIA, Amazonas: P.N.N Amacayacú (cerca boca Caño Mata-Mata), 1 o., 1.X.1988, F. Fernández col. (MIZA); Antioquia: Mpio San Luis (Río Claro, $\left.5^{\circ} 57^{\prime} \mathrm{N} 74^{\circ} 51^{\prime} \mathrm{O}, 430 \mathrm{~m}\right), 1$ o., 23.I.1998, D. Campos col. (IAVH); Cauca: Isla Gorgona, 1 o. (MIZA); Cundinamarca: Medina (Miralindo, 4³6'N 73²3’O, 1000 m), 1 o., II-III.1997, F. Escobar col. (IAVH); Meta: Bosque de Bavaria $(580 \mathrm{~m}), 1 \mathrm{o.}$ 20.X.191996, E. Palacios col. (IAVH); Nariño: Barbacoas (Tajadas, $1000 \mathrm{~m}$ ), 1 o.,1.VIII.1994, F. Escobar col., n 459 (IAVH); La Espriella (50 m, bosque), 2 o., VIII.1994, F. Escobar col., n 493 (IAVH); Tumaco, La Espriella $(500 \mathrm{~m}), 1$ o. 1.VIII.1994, F. Escobar col., n ${ }^{\circ} 589$ (IAVH); Barbacoas (Tajadas, 1000 m), 2 o., VIII.1994, F. Escobar col., n 363, 385 (IAVH); Quindío: Buenavista (vda. El Infierno, Finca Guadalajara, 4²2’36”N 7546’10”O, 1160 m), 1 o., 17.IX.1999, E. González col. (IAVH); Valle: Bajo Calima, 2 o., F. Castaño col.(MIZA); El Cerrito ( El Hatico,1000 m), 2 o., I. Armbrecht col. (en tronco, Pon-08) (IAVH); B. Calima $(50 \mathrm{~m}), 1$ o., 5.V.1996, H. Berrío col. (UDVC); Cali, 1 o., 22.I.1976, J. Lattke col. (MIZA). ECUADOR, Los Ríos: Río Palenque (2 km SSE, 0³5’ $\mathrm{S} 79^{\circ} 22^{\prime} \mathrm{O}$ Patricia Pilar, bosque pluvial, $160 \mathrm{~m}$,), 2 o., 15.VIII.1991, P. Ward col. (PSWC); Napo: Tena $\left(0^{\circ} 59^{\prime} \mathrm{S} 77^{\circ} 49^{\prime} \mathrm{O}\right.$, bosque pluvial, $520 \mathrm{~m}$ ), 1 o., 2.VIII.1991, P. Ward col. (PSWC); (25 km WSW Loreto, $0^{\circ} 44^{\prime} \mathrm{S} 77^{\circ} 31^{\prime} \mathrm{O}$, bosque pluvial, $\left.1050 \mathrm{~m},\right), 1 \mathrm{o}$. 8.VIII.1991, P. Ward col. (PSWC); Pichincha: Maquipucuna (5 $\mathrm{km}$ ESE Nanegal, $\left.0^{\circ} 07^{\prime} \mathrm{N} 78^{\circ} 38^{\prime} \mathrm{O}, 1250 \mathrm{~m}\right), 2$ o., $2 \mathrm{~m}$., 18.VIII.1991, P.S. Ward col. (MIZA); Sucua, 8 o., 2 m., 21.VII.1978, G. Umphrey col. (MIZA).

Comentarios: Los ejemplares de Barbacoas (Nariño) se diferencian de la versión usual de G. annulata por su mayor tamaño y oscuridad. Estas hormigas tienen el cuerpo negro y las patas castaños, salvo los meso- y metacoxas, trocánteres y la base de los fémures, que son castaño claros. Generalmente el cuerpo y las patas son castaños salvo la coloración castaño clara en los mesoy metacoxas, trocánteres y la base de los fémures y tibias. En otros aspectos corresponde bien a G. annulata, y restándole importancia a la coloración, aún llama la atención la diferencia de tamaño.

\section{Gnamptogenys banksi (Wheeler, 1930)}

Diagnosis. Con costillas finas; cabeza ancha con escapos longitudinalmente estríados; sutura mesometanotal sin impresión profunda, aunque visible; sin el surco mandibular largo y fino presente en $G$. laticephala.

Material examinado. ECUADOR, Guayas: $3 \mathrm{~km} \mathrm{~S}$ Bucay, 1 o. (MCZC).

\section{Gnamptogenys bispinosa (Emery, 1897)}

Diagnosis. Ojos semi-globosos, escapos largos, sobrepasando el margen verticeal; mandíbulas sin dientes; sutura promesonotal dorsalmente rompe la escultura, aunque no lateralmente; sutura mesometanotal profunda y ancha; cabeza, mesosoma y pospeciolo rugosos; ferrugínea.

Material examinado: COLOMBIA, Chocó: 10km SO San 
José del Palmar, Río Torito (Finca Los Guadales, $800 \mathrm{~m}$ ), 2 o., 4.VI.1978, C. Kugler col. (MIZA, FFIC); Cundinamarca: San Isidro de Tena (1800 m), 2 o., 28.III.1991, E. Palacio \& G. Amat col. (IAVH); Valle: Cuenca media Río Calima, Río Bravo, 1 o., 3.XI.1989, F. Castaño col. (IAVH); Lago Calima (1600 m), 1 o. (MIZA); Saladito (1900-2100 m) 1 o. (IAVH).

\section{Gnamptogenys bisulca Kempf \& Brown, 1968}

Diagnosis. Costillas longitudinales sobre el declive propodeal; espinas metacoxales en gancho y triangulares, no aciculares; costillas transversas bien definidas sobre el esterno pospeciolar; espiráculo propodeal tan bajo como la escultura que lo rodea, no elevado.

Material examinado. COLOMBIA, Chocó: 10 km SO San José del Palmar, Río Torito (Finca Los Guaduales, 800 m), 3 o., 4.VI.1978, C. Kugler col. (CUIC); Nariño: Barbacoas (Tajadas, 1000 m), 1 o., 1.IV.1994, F. Escobar col., n 438 (IAVH); P.N.N. Río Ñambí, Altaquer, Barbacoas, 1 o., X.1990, J. Bustos col. (IAVH); Ricaurte (La Planada, 1800 m), 1 o.,1.IV.1993, FP-67 (IAVH); Quindío: Filandia (Río Barbas, 443’N 75³9’O, 1655 m), 3 o., 5.II.2000, J. Sossa col. (IAVH); Risaralda: P.N.N. Ucumarí, 2 o., 1 r., 11.XII.1990, E. Palacio col. (MIZA); (La Suiza, 1800 m),1 o., IV.1995, S. Usma \& R. Aldana col. (IAVH); Valle: El Ensueño (1670 m), 1 o., 8.XII.1993, (IAVH); Calima, Río Bravo, 1 o., 17.IX.1989, F. Fernández col.; Lago Calima (1600 m), 6 o., 21.VI.1971, W.L. Brown col. (MIZA); Saladito (1900-2000 m), 1 o., 23.III.1967, R. Root \& W.L. Brown col. (MIZA); Represa Calima vía Loboguerrero (km 38, $1600 \mathrm{~m}$ ) 1 o., 14.III.1967, R. Root \& W.L. Brown col. (MIZA); Pichindé (SO Cali, 1570 m), 1 o., 22.III.1967, R. Root \& W.L. Brown col. (MIZA); Bosque Yotocó (1575 m), 5 o., 23.VI.1989, W. MacKay col. (MIZA). ECUADOR, Pichincha: 20-30 km ESE Allurquín vía Chiribago (1400-1800 m), 10 o., 1975, S. \& J. Peck col. (MIZA); Maquipucuna (5 km ESE Nanegal, $0^{\circ} 07^{\prime} \mathrm{N} 78^{\circ} 38^{\prime} \mathrm{O}$, 1250-1700 m), 12 o., P. Ward col. (MIZA); 3 km E Tandapi (1300 m), 15 o., 1 r., 1975, S. \& J. Peck col. (MIZA); 16 km SE Sto. Domingo de los Colorados $(680 \mathrm{~m}), 5$ o., 4 r., VI.1975, S. \& J. Peck col. (MIZA).

\section{Gnamptogenys brunnea Lattke, 1995}

Diagnosis. Similar a G. nigrivitrea. Dorso cefálico con costillaje longitudinal; margen verticeal cóncavo y ojos ligeramente detrás de la línea media; mandíbulas triangulares, con costillaje dorsal, márgenes apical y basal con franjas estrechas lisas y brillantes; margen pronotal anterior con 6-8 costillas transversas las cuales se arquean cerca al dorso y se convierten en costillas longitudinales; costillaje sobre el tergo gástrico 2 más fino que el segmento anterior; cuerpo café rojizo, patas amarillo-café.

Material examinado. COLOMBIA, Chocó: $10 \mathrm{~km}$ SO San José del Palmar, Río Torito (Finca Los Guadales, 760 m), 14 o., 1 r., 2.VI.1978, C. Kugler col. (MIZA); 2 o., 2.VI.1978, C. Kugler col. (MZSP); 2 o., 2.VI.1978, C. Kugler col. (USNM); Valle: Cuenca Media Río Calima (Río Azul, 750 m), 4 o., R. Aldana col. (IAVH, UNCB).

\section{Gnamptogenys caelata Kempf, 1967}

Diagnosis. Mandíbulas lateralmente arrugadas con el margen apical liso y pulido; escapos longitudinalmente arrugados; dorso del mesosoma sin suturas o surcos transversales; proceso subpeciolar semi-cuadrado; dentículos propodeales presentes.

Material examinado. COLOMBIA, Amazonas: $7 \mathrm{~km} \mathrm{~N}$ Leticia, 1 o., 10-25.II.1972, S. \& J. Peck col., B-230 (MIZA).

\section{Gnamptogenys concinna (Smith, 1858)}

Diagnosis. Cuerpo con estrías muy finas, propodeo con estriación transversal, declividad lisa medialmente; sutura promesonotal débilmente impresa o ausente; surco metanotal ancho y profundo; diente metacoxal ausente, como mucho con cresta o tubérculo bajo, poco conspicuo.

Material examinado. COLOMBIA, Amazonas: Araracuara, 1 o., G. Gangi col., n 150 (IAVH); P.N.N. Amacayacu (San Martín, 325'S 706'O, 150 m), 1 o., 29.XII.2000-6.I.2001, B. Amado col. (IAVH); Meta: R.N. La Macarena (580 m), 1 o., 1.X.1986, F. Fernández \& L. Schneider col. (FFIC); Nariño: La Espriella (50 m), 1 o., VIII.1994, F. Escobar col. (IAVH).

\section{Gnamptogenys continua Mayr, 1887}

Diagnosis. Mandíbulas de tendencia linear; surco metanotal impreso; cara en declive del propodeo con pequeños lóbulos superior laterales, mitad superior de cara con costillaje longitudinal e inferior con costillaje transverso; disco esternal del pospeciolo liso; cara anterior del nodo peciolar rugosa con algunas costillas transversas inferiores

Material examinado. COLOMBIA, Amazonas: Amacayacu, 4 o., II.1991, L. Mendoza col. (MIZA); Antioquia: Frontino (P.N.N. Orquídeas, Cabaña Venados, $900 \mathrm{~m}), 1$ o. 6.IV.1996, E. Palacio col. (EPIC); 1 m., 1 o., 1 r., 6.IV.1996, E. Palacio col. (MIZA); Mpio S. Luis (Río Claro, $5^{\circ} 57^{\prime} \mathrm{N}, 7^{\circ} 51^{\prime} \mathrm{O}$, 430m), 1 o., 20.I.1998, D. Campos col. (IAVH); Cundinamarca: Quebrada Susumuko $(980 \mathrm{~m}), 1$ o., 1 r. (MIZA); Magdalena: P.N.N. Tayrona (Pueblito, $360 \mathrm{~m}$ ), 4 o., 1 r., 24.II.1977, C. Kugler col. (MIZA); (11 $\left.{ }^{\circ} 20^{\prime} \mathrm{N} 74^{\circ} 2^{\prime} \mathrm{O}, 225 \mathrm{~m}\right), 2$ o., $12-$ 29.V.2000, R. Henriquez col. (FFIC, IAVH); Nariño: Orito (Territorio Kofán, $00^{\circ} 30^{\prime} \mathrm{N} \quad 77^{\circ} 13^{\prime} \mathrm{O}, 1430 \mathrm{~m}$ ), 2 o., E. González col. (IAVH); Valle: P.N.N. Farallones de Cali, (Embalse Alto Anchicayá, $\left.3^{\circ} 26^{\prime} \mathrm{N} 76^{\circ} 49^{\prime} \mathrm{O}, 650 \mathrm{~m}\right), 1$ o., 10.X.2000, S. Sarría col. (IAVH). ECUADOR, Pichincha: Tinalandia (16 km SE Santo Domingo de los Colorados, $680 \mathrm{~m}$ ), 2 o., S. Peck col. (MIZA).

\section{Gnamptogenys curvoclypeata Lattke, 1990}

Diagnosis. Cuerpo piceo; antenas, patas y coxas ferruginosas; sutura promesonotal muy débilmente impresa, visible solamente con ciertos ángulos de luz; meso y metacoxas lisas y brillantes sobre las caras mediana y basal lateral; metacoxas con lóbulos dorsales triangulares débilmente desarrollados.

Material examinado. COLOMBIA, Amazonas: $7 \mathrm{~km} \mathrm{~N}$ Leticia, 1 o., 10-25.II.1975, S. \& J. Peck col. (MIZA); Amacayacu, 2 o., 13.VIII.1990, F. Fernández col. (MIZA).

\section{Gnamptogenys ejuncida Lattke, 1995}

Diagnosis. Cara anterior pronotal con mas o menos 6 costillas transversales; proceso subpeciolar triangular, proyectado anteriormente; cara anterior del tergo pospeciolar con costillaje transversal hasta las dos terceras partes del lado dorsal, costillaje posterior longitudinal.

Material examinado. COLOMBIA, Antioquia: Mpio Frontino (P.N.N. Orquídeas, Norte Cabaña Venados, $900 \mathrm{~m}$ ), 6.IV.1996, E. Palacios col., PON 0082 (EPIC); Chocó: Río Atrato, 1 o., XI.1992, F. Fernández col. (IAVH); Putumayo: Mocoa (610 m), 1 o., 4.I.1977, C. Kugler col. (MIZA); Valle: Cuenca medio Río Calima ( 400 m), 1 o., 1992, M. Baena col., MLB-07 (IAVH); ca. Río Danubio (230 m), 1 o., 15.III.1996, H. Berrío col. (IAVH). 
Comentarios. Algunos de estos ejemplares podrían confundirse con G. pilosum por presentar pelos erguidos en la parte ventral de las coxas medias y posteriores. En G. pilosum el costillaje mesosomal posterior al pronoto es longitudinal y bien finito, mientras que en $G$. ejuncida es transversal y más grueso. En LATTKe (1995) esta diferencia en la esculturación no se usó en la clave por que en G. porcata, especie cercanamente emparentada, suele ser variable. Sin embargo en los diferentes ejemplares de G. pilosum y G. ejuncida que hemos podido ver, esta diferencia se ha mantenido estable. Falta determinar el grado de variabilidad en la extensión posterior del costillaje transversal sobre el dorso pospeciolar de esta especie. En los ejemplares citados ésta se mantienen principalmente longitudinal.

\section{Gnamptogenys enodis sp. nov.}

(Figs. 1, 2)

Diagnosis. Mandíbulas triangulares, alargadas, ojos semiesféricos; escapos mayoritariamente lisos con algunas arrugas longitudinales; propodeo con franja mediana de costillaje longitudinal rodeado por costillaje circular; dientes propodeales breves; pecíolo sin nudo, más largo que ancho; dientes metacoxales aciculares.

Material- tipo. Obrera holotipo. COLOMBIA, Valle del Cauca, P.N.N. Farallones de Cali (Anchicayá, $3^{\circ} 26^{\prime} \mathrm{N} 76^{\circ} 48^{\prime} \mathrm{W}$, 730 m), Winkler, 17-20.VII.2001, S. Sarria col., M. 2849 (IAVH) Paratipos:(1) COLOMBIA, Valle del Cauca, P.N.N. Farallones de Cali (Anchicayá, 32 $26^{\prime} \mathrm{N} 76^{\circ} 48^{\prime} \mathrm{W}, 900 \mathrm{~m}$ ), una obrera, 1618.I.2001, S. Sarria col., winkler, M. 1566 (MIZA); (2) (730 m), 1 reina y 3 obreras, 21-23.I.2001, S. Sarria col., winkler, $\mathrm{n}^{\circ} 1571$ (IAVH y MZSP)

Obrera. Cabeza en vista dorsal semi-cuadrada, lateralmente suavemente convexo, perfil posterior ligeramente cóncavo; perfil anterior de la lamina del clípeo recta, lateralmente redondeada; ojos protuberantes, semiesféricos. Del margen anterior del clípeo salen unos 10 pelos conspicuos, largos, extendiéndose hacia delante hasta la mitad de la longitud mandibular. Mandíbulas triangulares (fig. 1), alargadas, con una lamina traslucida en el perfil interior, separada por un diente del perfil masticador, el cual tiene 9-7 dientecitos irregularmente espaciados entre sí; superficie dorsal con estriación longitudinal; perfil externo con una suave concavidad. Escapos principalmente lisos y pulidos, con algunas arrugas longitudinales, sobrepasando el perfil de vértice por más de dos diámetros apicales, con algunos pelos semi-erguidos; segmentos del flagelo mas largos que ancho. Cabeza con fuerte costillaje longitudinal hasta el vértice, 19-16 costillas presentes entre las carenas frontales; vértice bordeado posteriormente por prominente lámina trasversal.

Mesosoma en vista lateral con perfil dorsal plano y continuo, sin interrupción por suturas impresas, perfiles dorso-anterior y dorso-posterior convexos. Costillaje mayoritariamente longitudinal, superficie lateral del pronoto con costillaje oblicuo, ascendiendo posteriormente; anepisterno separado por suturas, sutura mesopleural presente, katepisterno separado de metapleura por sutura mesometapleural bien definida; lado del propodeo con arrugas. Lóbulo metapleural bien definido, proyectándose encima de metacoxa; espiráculo propodeal redondo, circunscrito por anillo elevado de cutícula, diente propodeal triangular, su longitud entre 2-3 veces el ancho de su base. Borde anterior de pronoto con 9-6 costillas transversales; dorso pronotal con franja media de costillaje longitudinal circunscrito por costillaje circular que forma franja de 5 8 costillas transversales contiguas a surco metanotal estrecho pero bien definido; sutura promesonotal apenas se divisa como breve hendidura trasversal. Cutícula del propodeo mayoritariamente arrugada y zigzagueante salvo franja dorsal de costillaje longitudinal; cara en declive del propodeo pasa a costillas trasversas, poco definidas.

Pecíolo con costillaje trasversal, en vista lateral con perfil dorsal suavemente convexo (fig. 2), sin nodo bien definido; proceso ventral forma breve lóbulo anterior. En vista dorsal un poco más largo que ancho, lados convergentes anteriormente con lóbulos anterolaterales. Proceso del primer esterno proyectándose como lóbulo redondeado. Tergos gastrales con costillaje longitudinal y uniforme; esterno pospeciolar con costillaje transversal menos definido. Coxas con costillaje trasversal; tibias y fémures lisos y pulidos; superficie dorsal protarsal con arrugas longitudinales, base del protarso opuesto al espolón con una sola seta gruesa; espina metacoxal larga y acicular. Cuerpo marrón oscuro; antenas, mandíbulas, y patas marrón ferruginoso.

Medidas del holotipo (paratipo): AC 0.91 (0.95), LC 0.98 (1.01), LE 1.00 (1.10), DO 0.19 (0.20), LM 0.70 (0.79), LW 1.65(1.79); IC 0.93 (0.94), IE 1.10 (1.21), IM 0.76 (0.81), IO 0.21 (21).

Reina y macho. Desconocidos.

Comentarios. Esta especie se asemeja mucho a $G$. mecotyle y la clave en LATTKE (1995) lleva a dicha conclusión. G. enodis se diferencia de G. mecotyle, y de otras Gnamptogenys, a primera vista por la aparente ausencia de un nudo peciolar. En vista lateral el pecíolo forma una continua y suave convexidad que no permite definir un nudo como estructura aparte del pecíolo. En $G$. mecotyle el nudo esta reducido pero sigue siendo claramente diferenciado y el proceso ventral tiene forma rectangular con un ángulo anterior y otro posterior, y el perfil ventral algo cóncavo, mientras que en $G$. enodis el proceso es un modesto lóbulo. En G. mecotyle el costillaje pronotal es totalmente longitudinal mientras que en $G$. enodis presenta una serie de costillas en forma circular que rodena una pequeña franja de costillaje longitudinal. En una vista dorsal el pecíolo es relativamente mas alargado y los escapos tienden a ser mas lisos y con estrías menos definidas en G. enodis que en G. mecotyle. Se presume que por su cercanía a G. mecotyle, esta nueva especie también es un cazador especializado de diplópodos.

Etimología. El nombre de la especie se deriva del Latin, enodis, libre de nudos y se refiere a la carencia del nudo peciolar en esta especie.

\section{Gnamptogenys ericae Forel, 1912}

Diagnosis. Mandíbulas semi-triangulares; dorso mesosomal posterior y propodeo con costillaje longitudinal, suturas transversas difícilmente visibles 


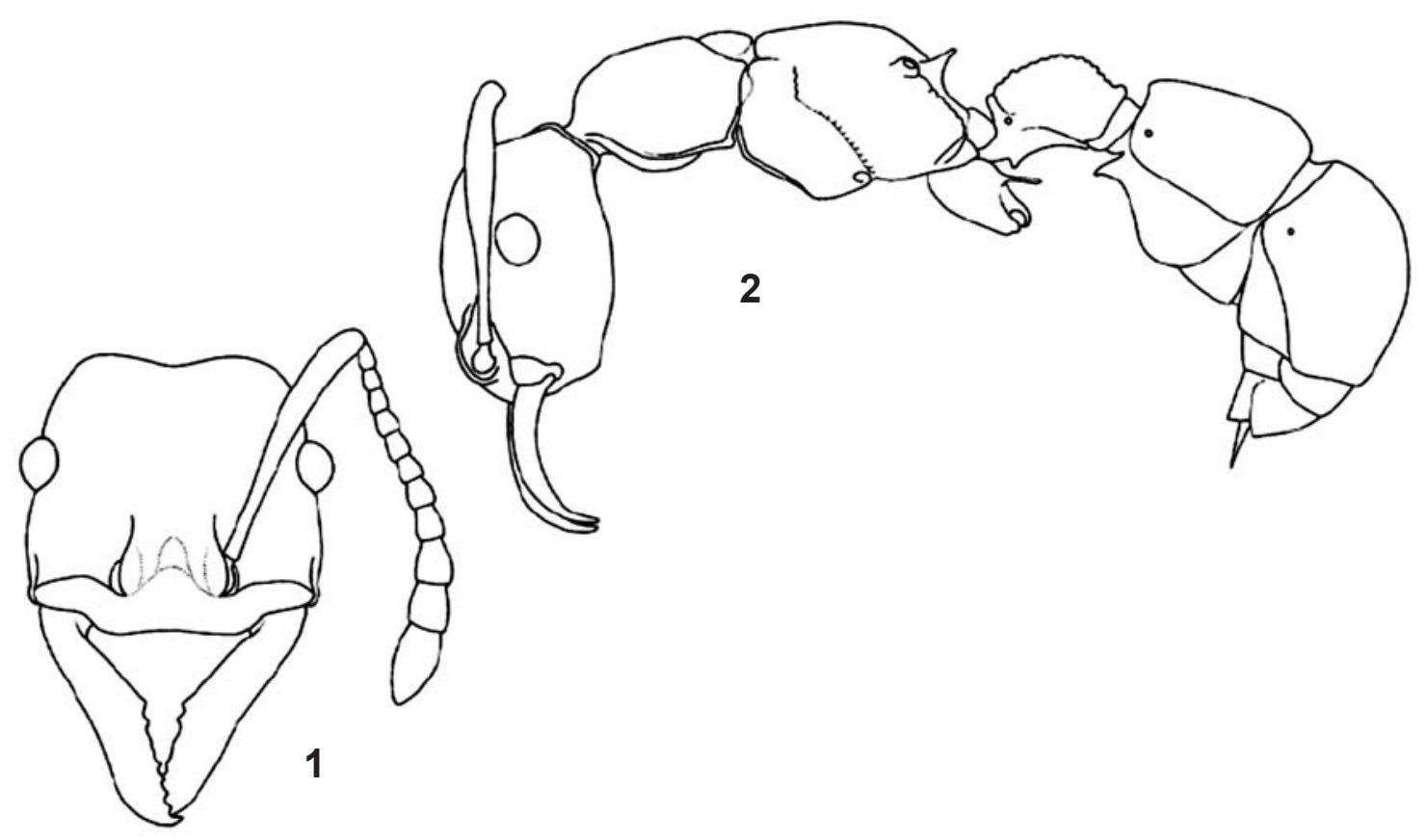

Figs. 1, 2. Obrera de Gnamptogenys enodis, sp. nov., parátipo (Colombia): 1, Cabeza, frontal; 2, cuerpo (se omiten las patas), lateral (AC $0,95 \mathrm{~mm}$; LW $1,65 \mathrm{~mm}$ ).

bajo ciertos ángulos de luz; dientes metacoxales pequeños; negras, antenas, patas y mandíbulas café a café oscuro.

Material examinado. COLOMBIA, Casanare: 3 o., XII.1991, M. Castro col. (MIZA); Guajira: Serranía de Macuira (7 km S Nazareth, 70-200 m), 1 o. (MCZC); Magdalena: Minca $(610 \mathrm{~m}), 1$ o. (MIZA); P.N.N. Tayrona (Zaino, $50 \mathrm{~m}), 1 \mathrm{r}$, $\left.11^{\circ} 20^{\prime} \mathrm{N} 74^{\circ} 02^{\prime} \mathrm{O}\right), 28$.VII-14.VIII.2000, R. Henriquez col. (IAVH); Meta: San Juan de Arama (914 m), 2 o. (FFIC); La Macarena (Rio Guayabero, 260 m), 1 o., 15.I.1977, C. Kugler col. (MIZA); (La Curria, 580 m), 1 o., VII.1990, E. Palacio col. (EPIC); San Juan de Arama, 1 o., 25-26.XII.1976, W. \& E. MacKay col. (MIZA); Sucre: Estación de Primates Inderena, 1 o., VII.1989 (MIZA).

\section{Gnamptogenys extra Lattke, 1995}

Diagnosis. Vértice con 2-3 costillas transversales; superficie anterior pronotal con 5-7 costillas transversales; nudo peciolar en vista lateral con un margen anterodorsal ampliamente convexo, ligeramente sobresaliendo encima del margen posterior, margen posterior relativemente recto; proceso subpeciolar semicuadrado. Apice del nodo peciolar bastante puntudo, no tan extremamente como en G. acuta. Nodo más robusto y semi-cuadrado (en vista lateral) que en otras especies del subgrupo porcata.

Material examinado. COLOMBIA, Cauca: Río Abuya, 1 o., 1994, A. Feijo col. (IAVH); Chocó: Lloró (Peñalosa), 3 o., 26.III.1988 (BMNH, LACM, MIZA); Valle: Bajo Anchicayá (270 m), 1 o., 1994, D. \& L. col., en oso hormiguero (IAVH); R. F. Escalerete $(80 \mathrm{~m}), 1$ o., XI.1995, S. Usma \& R. Aldana col. (IAVH); Andalucía (Quebrada El Naranjo), 1 o., V.1990, M. Baena col. (UDVC). ECUADOR, Pichincha: Tinalandia (16 km SE Santo Domingo de los Colorados), 2 o., VI.1976, S. \& J. Peck col. (MIZA).

\section{Gnamptogenys fernandezi Lattke, 1990}

Diagnosis. Mandíbulas triangulares con el dorso liso y brillante; lamina anterior del clípeo convexa; sutura promesonotal ligeramente impresa y sutura mesometanotal ausente; peciolo bajo, alargado, posterodorsalmente con punta roma; diente metacoxal ausente.

Material examinado. COLOMBIA, Valle: $(50 \mathrm{~m}), 1 \mathrm{o}$. (MCZC); Merizalde, 1 o., 3.XII.1988, M. Mendoza col. (IAVH). ECUADOR, Morora: Santiago (Los Tayos), 1 o. (MCZC).

\section{Gnamptogenys gentryi Lattke, 1995}

Diagnosis. Surco metanotal más profundo que sutura promesonotal; gáster en su mayoría liso y pulido.

Material examinado. COLOMBIA, Nariño: Altaquer (1350 m), 1 o., 23.VII.1993, C. Bustos col. (IAVH); Valle: Pance (Hato Viejo, 2250 m), 1 o., J. B. Hillaire col. (MIZA).

\section{Gnamptogenys haenschi Emery, 1902}

Diagnosis. Margen verticeal cóncavo en vista frontal; mandíbulas con costillaje longitudinal; cara en declive del propodeo con estrías longitudinales superiormente, basalmente con arrugas transversas; nodo peciolar transverso; esterno pospeciolar en su mayoría transversamente estrigulado; sin diente metacoxal.

Material examinado. COLOMBIA, Antioquia: Mpio S. Luis (Río Claro, 557’’ , 7451’’O, 430 m), 1 o., 20.I.1998, D Campos col. (IAVH); Cauca: Isla Gorgona, 1 o. (MIZA); Chocó: Ríosucio (La Balsa, 70 m), X.1994, L. Mendoza col.; Guajira: Quebrada Guachoque, 1 o. (MIZA); Magdalena: $4 \mathrm{~km} \mathrm{~N} \mathrm{San}$ Pablo (550 m), 1 o., (MIZA); Sierra Nevada de Santa Marta (Transecto Buritica - La Cumbre, 900 m),1 o., VII-IX.1997, T. v.d. Hammen col. (UNCB); Valle: San Cipriano, 1 o., VII.1989, 
M. Baena col.(IAVH); Cuenca Media Río Calima (Río Azul, 550 m), 3 o., IV.1994, R. Aldana col. (IAVH). ECUADOR, Napo: Puerto Misahualli (350 m), 1 o. (MIZA).

\section{Gnamptogenys hartmanni (Wheeler, 1915)}

Diagnosis. Esquinas superolaterales de la cara en declive del propodeo con lóbulos o carenas pequeñas; mandíbulas triangulares a semi-triangulares; surco metanotal vagamente impreso y cara posterior del nodo con costillaje longitudinal; dorso metacoxal lobulado; proceso subpeciolar semi-cuadrado.

Material examinado. COLOMBIA, Antioquia: Mpio San Luis (Río Claro, 557’ N 7451’O, 430 m), 1 o., 20.I.1998, D. Campos col. (IAVH).

\section{Gnamptogenys horni Santschi, 1929}

Diagnosis. Mandíbulas semi-triangulares; dorso del nodo del pecíolo con costillas longitudinales posteromedianas, anteriormente transversamente arqueadas; costillaje en forma de "v" sobre la cara en declive del propodeo; cuerpo café oscuro; patas y antenas ferruginosas.

Material examinado. COLOMBIA, Antioquia: Mpio S. Luis (Río Claro, $5^{\circ} 57^{\prime} \mathrm{N} 74^{\circ} 51^{\prime} \mathrm{O}, 430 \mathrm{~m}$ ), 1 o., 20.I.1998, D. Campos col. (IAVH); Chocó: Río Napipí, 1 o. (MIZA); Guaviare: P.N.N. Nukak Maku (Río Inírida, $180 \mathrm{~m}$ ), 2 o., 2.II.1996, F. Fernández col. (IAVH); Meta: R.N.N. La Macarena (Caño La Curía, 580 m), 1 o., VII.1990, E. Palacio col. (EPIC); (La Curia, $580 \mathrm{~m}$ ), 6 o., VII.1990, E. Palacios col. (MIZA); Bosque de Bavaria $(580 \mathrm{~m}), 1$ o., 20.XI.1996, E. Palacio col. (EPIC); Mpio Mesetas, (Inspección de la Uribe, $750 \mathrm{~m}$ ), 5 o. 1.XII.1997, F. Fernández col. (MIZA); Nariño: Territorio Kofán $\left(00^{\circ} 30^{\prime} \mathrm{N} 77^{\circ} 13^{\prime} \mathrm{O}, 1430 \mathrm{~m}\right), 1$ o., 25.IX.1998, E. González col. (IAVH); Quianga, 1 o., 21.IX.1989 (MIZA); Risaralda: Pueblo Rico (Santa Cecilia, 550 m), 1 o., 26.II.1992, E. Palacio col. (EPIC); Valle: Andalucia (Quebrada El Naranjo), 1 o., V.1990, M. Baena col. (IAVH); R. F. Escalerete $(80 \mathrm{~m}), 1$ o., VIII.1995, S. Usma \& R. Aldana col. (UDVC); Zarzal, bosque del medio, 1 o., 950 m, 19.I.1995, I. Armbrecht col. (IAVH); P.N.N. Farallones de Cali (Embalse Alto Anchicayá, $3^{\circ} 26^{\prime} \mathrm{N} 76^{\circ} 48^{\prime} \mathrm{O}, 650 \mathrm{~m}$ ), 2 o., 10.X.2000, S. Sarría col. (IAVH). ECUADOR, Esmeraldas: $48 \mathrm{~km} \mathrm{~S}$ Atacames, 1 o. (MIZA); Pichincha: Estación Río Palenque (Sucua), 1 o. (MIZA); Morona: Santiago, Los Tayos, 1 o. (MIZA); Napo: Tena, $\left.0^{\circ} 59^{\prime} \mathrm{S} 77^{\circ} 49^{\prime} \mathrm{O}, 520 \mathrm{~m}\right), 1$ o., 2.VIII.1991, bosque pluvial (MIZA).

\section{Gnamptogenys ingeborgae Brown, 1992}

Diagnosis. Mandíbula con margen masticatorio denticulado; sutura promesonotal presente, al menos parcialmente, y costillaje longitudinal sobre el dorso del propodeo y tergo pospeciolar frecuentemente mal definido a borrado.

Material examinado. COLOMBIA, Cundinamarca: vía Bogotá-Villavicencio, km $79(1100 \mathrm{~m}), 1$ o. (MCZC), 2 o., $1 \mathrm{~m}$. (MIZA).

\section{Gnamptogenys interrupta Mayr, 1887}

Diagnosis. Mandíbulas semi-lineares; dorso cefálico, mesosoma y primer tergo gastral con costillaje longitudinal; tergo gastral 2 liso; pleura con parches lisos; diente metacoxal ausente; cuerpo café rojizo, patas y antenas ferruginosas.

Material examinado. COLOMBIA, Magdalena: $3 \mathrm{~km}$ SE Minca $(1050 \mathrm{~m}), 1$ o. (MIZA); Valle, Puerto Merizalde, 1 o., 10 m. (MIZA)

\section{Gnamptogenys kempfi Lenko, 1964}

Diagnosis. Mandíbulas semi-triangulares, dorsalmente lisas y brillantes; costillaje transverso sobre dorso mesosomal y nodo, sin suturas transversas aparentes sobre el dorso mesosomal; espiráculo propodeal grande y diente metacoxal presente.

Material examinado. COLOMBIA, Amazonas: $7 \mathrm{~km} \mathrm{~N}$ Leticia, 1 o. (MCZC).

\section{Gnamptogenys lanei Kempf, 1960}

Diagnosis. Lámina del clípeo ligeramente cóncavo medialmente, sutura promesonotal reducida, dentículos propodeales pequeños, nudo peciolar más largo que ancho con costillaje transversal sobre dorso.

Material examinado. COLOMBIA, Amazonas: P.N.N Amacayacu $\left(3^{\circ} 23^{\prime} \mathrm{S}, 70^{\circ} 6^{\prime} \mathrm{W}, 150 \mathrm{~m}\right), 1$ r., 19-26.VI.2000, A Parenti col. (IAVH).

\section{Gnamptogenys laticephala Lattke, 1995}

Diagnosis. Los dos lóbulos entre las fosas antenales y la lamina clipeal distinguen a esta especie de las demás del género en el Nuevo Mundo.

Material examinado. ECUADOR, Guayas: $3 \mathrm{~km} \mathrm{~S}$ Bucay, 1 o., 24.VII.1973, W.L. Brown col. (MCZC).

\section{Gnamptogenys mecotyle Brown, 1958}

Diagnosis. Sutura promesonotal y surco metanotal impresos pero sin romper la escultura; abertura del espiráculo propodeal grande y redondeada, recta debajo del diente; diente metacoxal largo y agudo; carena occipital ancha y visible en vista frontal; lamina clipeal anterior lateralmente redondeada y anteriormente recta a ampliamente convexa.

Material examinado. COLOMBIA, Amazonas: P.N.N. Amacayacu (Río Mata-Mata), 1 r., III.1988, M. Kelsey col. (MIZA); $7 \mathrm{~km} \mathrm{~N}$ Leticia, 7 o. (MIZA); Antioquia: Providencia, Estación Biológica, 13 o. (MIZA); Chocó: Serranía de Baudó (Camino de Yupe, 500-700 m), 1 o. (MIZA). ECUADOR, Napo: Jatun Sacha ( $7 \mathrm{~km}$ ESE Pto. Misahualli, $1^{\circ} 04^{\prime} \mathrm{S} 77^{\circ} 37^{\prime} \mathrm{O}, 400$ m), 5.VII.1991, bosque pluvial, 1 o. (MIZA).

\section{Gnamptogenys mina (Brown, 1957)}

Diagnosis. Dorso mandibular liso y brillante y ápice del escapo sobrepasando el margen verticeal; cara en declive del propodeo plana y separada de la cara dorsal por un ángulo agudo; espiráculos propodeales elevados hacia el ápice de los tubérculos cilíndricos.

Material examinado. COLOMBIA, Nariño: Territorio Kofán (Orito, $00^{\circ} 30^{\prime} \mathrm{N} 77^{\circ}$ 13’O, 700 m), 4 o., 28.IX.1998, E. González col. (IAVH); Putumayo: Villa Garzón, 1 o. (MCZC). ECUADOR, Napo: Jatun Sacha (7 km ESE Pto. Misahualli, $1^{\circ} 04$ 's $77^{\circ} 37^{\prime} \mathrm{O}, 400 \mathrm{~m}$ ), 1 o., 5.VII.1991, bosque pluvial (MIZA).

\section{Gnamptogenys minuta (Emery 1896)}

Diagnosis. Esculturación de aspecto opaco y finamente granuloso; mandíbulas y antenas castañas, 
estriadas y brillantes; espiráculos propodeales generalmente elevados, formando tubitos.

Material examinado. COLOMBIA, Antioquia: Mpio S. Luis (Río Claro, 557’ N, 7451’’O, 430 m), 1 o., 21.I.1998, D. Campos col. (IAVH); Chocó: Mumbu (Quebrada Mumbu, 500 m), 1 o., X.1991, E. Florez col. (IAVH); Magdalena: El Campano $\left(11^{\circ} 07^{\prime} \mathrm{N} 74^{\circ} 06^{\prime} \mathrm{O}, 1300 \mathrm{~m}\right), 1$ o., 13.VII.1985, P. Ward col. (MIZA); Cabo - Pueblito (200 m), 1 o., 2.IV.1977, C. Kugler col. (CUIC). ECUADOR, Pichincha: $47 \mathrm{~km} \mathrm{~S}$ Santo Domingo de los Colorados (Estación Río Palenque, 215 m), 3 o., 23.V.1975, S. \& J. Peck col. (MIZA).

\section{Gnamptogenys moelleri (Forel, 1921)}

Diagnosis. Costillaje ligeramente tosco; pronoto con costillaje concéntrico; cara mesosomal posterodorsal con costillaje longitudinal; proceso subpeciolar semicuadrado, algunas veces anteriormente proyectándose en forma de lóbulo.

Material examinado. COLOMBIA, Amazonas: $7 \mathrm{~km} \mathrm{~N}$ Leticia, 1 o. (MIZA); Chocó: Finca Los Guaduales (800 m), 2 o. (MIZA); Cundinamarca: Medina (Miralindo, $4^{\circ} 36^{\prime} \mathrm{N} 73^{\circ} 23^{\prime} \mathrm{O}$, 1000 m), 1 o., II-III.1997, F. Escobar col. (IAVH); Meta: Reserva La Macarena (580 m), 2 o. (FFIC); (Río Guayabero), 1 o. (UNCB), 5 o. (MIZA); P.N.N. La Macarena, 1 o., 1987, F. Fernández col. (MIZA); 2 o., 8.VIII.1991, E. Palacio col. (MIZA); Nariño: 28 km S Mocoa $(510 \mathrm{~m}), 1$ o. (UNCB); Putumayo: $5 \mathrm{~km} \mathrm{~S}$ Mocoa (610 m), 8 o., 3.I.1977, C. Kugler col. (MIZA). ECUADOR, Napo: Limoncocha, 3 o., 18.V.1976, B-348, S. \& J. Peck col. (MIZA).

\section{Gnamptogenys mordax (Smith, 1858)}

Diagnosis. Mandíbulas semi-triangulares; cara en declive del propodeo con costillaje transverso; segundo tergo gastral puede variar desde liso a costillaje longitudinal o costillado-rugoso; cuerpo muy oscuro, café, casi negro; patas café oscuro; individuos más pequeños pueden confundirse con G. continua.

Material examinado. COLOMBIA, Amazonas: P.N.N. Amacayacu (Caño Mata-Mata, $3^{\circ} 48^{\prime} \mathrm{S} 70^{\circ} 20^{\prime} \mathrm{O}$ ), 1 r., 2 o., 5.IX.1997, F. Fernández col. (IAVH); Antioquia: Mpio Frontinos (P.N.N. Orquídeas, Norte Cabaña, Venados, $900 \mathrm{~m}$ ), 1 o., 6.IV.1996, E Palacio col. (EPIC); Chocó: P.N.N. Utria, 0601'N $77^{\circ} 21^{\prime} \mathrm{O}, \quad 4$ o., 7.VI.1991, M. Baena col. (IAVH); Cundinamarca: Medina Miralindo (Qda. Ardita, $1450 \mathrm{~m}$ ), 1 o., 28.I.1997, F. Escobar col. (IAVH); Risaralda: Sta. Cecilia, 23.X.1992, 1 r. (MIZA); Santander: Charalá Virolín Cost. (E1 Fara, $06^{\circ} 06^{\prime} \mathrm{N} 73^{\circ} 13^{\prime} \mathrm{O}, 1800$ m), 1 o., 29.III.1999, E. González col. (IAVH); Valle: P.N.N. Farallones de Cali $\left(3^{\circ} 26^{\prime} \mathrm{N} 76^{\circ} 48^{\prime} \mathrm{W}\right.$, 900 m), 1 r., 25.X-8.XI.2000, S. Sarria col. (IAVH). ECUADOR, Napo: Jatun Sacha ( $7 \mathrm{~km}$ ESE Pto. Misahualli, $1^{\circ} 04^{\prime} \mathrm{S} 77^{\circ} 37^{\prime} \mathrm{O}$, $400 \mathrm{~m}), 1$ o., 5-VII-1991, bosque pluvial (MIZA); Pichincha: Sucua $(1500 \mathrm{~m}), 7$ o., 23.VII.1987, G. Umphrey col. (MIZA).

\section{Gnamptogenys nigrivitrea Lattke, 1995}

Diagnosis. Cabeza en vista frontal alargada y de lados aproximadamente paralelos; lámina del clipeo con un lóbulo mediano. Nudo peciolar con un margen anterodorsal convexo, formando un punta roma posterior que sobresale encima del margen posterior.

Material examinado. COLOMBIA, Caldas: Aguadas (Los Naranjos, 2220 m), 1 o., XI.1994, C. Sarmiento col. (IAVH); Caquetá: P.N.N. Picacho $\left(2^{\circ} 48^{\prime} \mathrm{N} 74^{\circ} 51^{\prime} \mathrm{O}, 1800 \mathrm{~m}\right), 1$ o., XI.1997, F. Escobar col. (UNCB); Cundinamarca: Medina (Miralindo, $4^{\circ} 35^{\prime} \mathrm{N} 73^{\circ} 23^{\circ} \mathrm{O}, 1000 \mathrm{~m}$ ), 1 o., III-IV.1994, F. Escobar col. (UNCB); Risaralda: La Suiza (1800 m), 1 o., IV.1995, Usma, y Aldana col. (IAVH); P.N.N. Ucumarí (1800 m), 1 o., V.1994, R. Aldana col. (IAVH); Nariño: La Planada $(1800 \mathrm{~m}), 1$ o., 18.VII.1995, N. Crystal Arens col. (IAVH); Barbacoas, Ñambí (1350 m), 1 o., F. Escobar col. (IAVH); Ricaurte (Reserva La Planada, 1800 m), 2 o., 1.IV.1993, M. L. Baena (IAVH); Ricaurte (Reserva La Planada, $1^{\circ} 15^{\prime} \mathrm{N} 78^{\circ} 15^{\prime} \mathrm{W}, 1850$ 1885 m), 7 o., 2-6.I.2001, 16.VII-2.VIII.2000, G. Oliva col. (IAVH); Putumayo: Mocoa $\left(1^{\circ} 08^{\prime} \mathrm{N} 76^{\circ} 38^{\prime} \mathrm{O}, 1800 \mathrm{~m}\right), 1 \mathrm{o}$, I.1999, E. González col. (IAVH); Risaralda: Ucumarí (La Suiza, 1830 m), 1 o., III.1992, E. Palacio col. (MIZA); Valle: Reserva Forestal de Yotocó (42 km NNE Cali, 1100-1500 m), 2 o., 8.I.1984, W. MacKay col. (MIZA, LACM); Bosque de Yotocó, 1 o., 6.I.1984, W. MacKay col. (MIZA); $(1575$ m), 2 o. 1 r., 23.VI.1989, W. MacKay col. (MIZA); P.N.N. Los Farallones (Pico de Aguila, 1600 m), 2 o., IV.16.1992, M. Gamboa col. (MIZA); Calima (Río Bravo), 1 o., 17.IX.1989 (MIZA); cerca Saladito (1900 m), 1 o., 1978 (MIZA).

Comentarios. Los ejemplares de Aguadas (Caldas) tienen el costillaje cuticular con finas corrugaciones, al contrario de lo que frecuentemente se ve en esta especie donde el costillaje es liso, de aspecto casi esmaltado.

\section{Gnamptogenys perspicax Kempf \& Brown, 1970}

Diagnosis. Ojos ligeramente detrás de la línea media cefálica; escapo longitudinalmente estríado con pilosidad abundante; nodo del pecíolo más o menos continuamente convexo; esterno del pospeciolo con costillaje o arrugas transversas.

Material examinado. COLOMBIA, Cundinamarca: San Isidro de Tena (Laguna de Pedro Palo), 1800m, 1 o., 12.V.1990, G. D. Amat col. (IAVH); Valle: Pichindé (1600 m), 1 o., 1973, (MIZA). ECUADOR, Pichincha: Estación Río Palenque, 1 o., 29.VII.1978, G. Umphrey col. (MCZC); 13 o., 2 r., 29.VII.1978, G. Umphrey col. (MIZA).

\section{Gnamptogenys pilosa Lattke, 1995}

Diagnosis. Vértice con 1-2 estrias transversales; lado dorsal del mesosoma longitudinalmente estriado; tergo del pospeciolo con estrías transversales anterodorsales hasta la dos tercera parte, posteriormente longitudinales.

Material examinado. COLOMBIA, Antioquia: P.N.N. Orquideas (Norte Cabaña Venados, 900 m), 1 o., 6.V.1996, E. Palacio col. (EPIC); (Urruao, 1430 m), 1 o., 4.IV.1996, E. Palacio col. (EPIC); Chocó: Guarato (Qda. Cuadralito), 2 o., 29.II., F. Fernández col. (MIZA); Quindío: Buenavista (Fca. Ceilán, 4²1'30'N 7547’05”'O, 1100 m), 3 o., 15.XI.1999, E. González y J. Sossa col. (IAVH); Valle: ca. Río Danubio $(230 \mathrm{~m}), 1$ o., 15.III.1996, H. Berrio col. (UDVC); Calima Medio (550 m), 1 o., R. Aldana col. (IAVH); Cuenca Media Río Calima (Río Azul, 750 m), 3 o., 1.VIII.1994, R. Aldana col. (IAVH); Calima (Cañón El Pital, F. Castaño, 750 m), 1 o., 1.VIII.1994, R. Aldana col. (BMNH); 1 o., 1.VIII.1994, R. Aldana col. (LACM); 1 o., 1.VIII.1994, R. Aldana col. (MCZC); 1 o., 1.VIII.1994, R. Aldana col. (MIZA); Quebrada Aguabonita, 1 o., 1.V.1992, M. L. Baena col. (IAVH)

\section{Gnamptogenys pleurodon (Emery, 1896)}

Diagnosis. Pronoto con costillaje concéntrico; mesonoto con costillaje longitudinal, algunas veces rodeado por costillas concéntricas; cara anterior del pospeciolo con 3 a 4 costillas transversas y cara dorsal con costillaje longitudinal; diente metacoxal acicular; cuerpo café oscuro, patas café.

Material examinado. COLOMBIA, Amazonas: $7 \mathrm{~km} \mathrm{~N}$ 
Leticia; 1 o. (MIZA), Guaviare: R.N.N. Nukak Makú (Río Inírida, Caño Cocuy, 180 m), 1 o., 5.II.1996, F. Fernández col. (IAVH); Putumayo: $5 \mathrm{~km}$ S Mocoa $(610 \mathrm{~m}), 1$ o. 3.I.1977, C. Kugler col. (MIZA); Valle: Bajo Anchicayá (270 m), 1 o.,1994, en oso hormiguero (IAVH). ECUADOR, Napo: Sushijindi (Tena, $400 \mathrm{~m}), 1$ o. (MIZA); Jatún Sacha (7 km ESE Pto. Misahualli, $1^{\circ} 04^{\prime} \mathrm{S} 77^{\circ} 37^{\prime} \mathrm{O}$, bosque pluvial, $400 \mathrm{~m}$ ), 4 o., 2-4.VII.1991 (MIZA)

\section{Gnamptogenys porcata (Emery, 1896)}

Diagnosis. Costillaje longitudinal media sobre el pronoto inscrito anteriormente por costillaje transverso; escultura mesosomal posterodorsal variable: ovaloides concéntricos con orientación longitudinal o transversa, o con costillas longitudinales inscritas dentro de los concéntricos; cuerpo píceo con patas ferruginosas.

Material examinado. COLOMBIA, Antioquia, Río Porce $(1020 \mathrm{~m}), 1$ o., N. Weber col. (MCZC); Antioquia: P.N.N. Orquideas (Urruao, $1430 \mathrm{~m}$ ), 2 o., 4.IV.1996, E. Palacio col. (EPIC); Cundinamarca: Tena (Laguna Pedro Palo, $2100 \mathrm{~m}$ ) 1 o., 15.X.1995, D. Campos col. (IAVH); arriba de Tena (1300$1600 \mathrm{~m}), 1$ o. (IAVH); Nariño: Ricaurte, (Reserva La Planada, 1800 m), 1 o., 1.III.1994, F. Escobar col. (IAVH); Río Ñambí (vda. Patio, $560 \mathrm{~m}$ ), 1 o., J. Bustos col., N-102 (IAVH); Barbacoas (Tajadas, $600 \mathrm{~m}$ ), 1 o., 1994, F. Escobar col. (IAVH); (Tajadas, $1000 \mathrm{~m}$ ), 1 o., E. Palacios col. (EPIC); Norte de Santander: P.N.N. Tamá (Alto de Herrera, vda. Diamante, $7^{\circ} 66^{\prime} \mathrm{N} 72^{\circ} 13^{\prime} \mathrm{O}, 1250 \mathrm{~m}$ ), 1 o., E. González col. (IAVH); Valle: Calima Medio (550 m), 1 o., IV.1994, R. Aldana col., (IAVH); Calima (Río Bravo, 3.IX.1989, 1 o. (MIZA); 3,2 km arriba Río Agua Clara (antigua vía Cali-Buenaventura), 2 o., R. Root \& W.L. Brown col. (MIZA); Río San Juan (cerca a Queremal, $1300 \mathrm{~m}), 1$ o. (MIZA); Buenaventura $(650 \mathrm{~m}), 1$ o. (UDVC); San Francisco, 2 o., 13.XII.1988, M. Baena col. (IAVH). ECUADOR, Pichincha: Tinalandia, (16 km SE Santo Domingo de los Colorados, $680 \mathrm{~m}$ ), 1 o., S. \& J. Peck col. (MIZA); Maquipucuna ( $5 \mathrm{~km}$ ESE Nanegal, $\left.0^{\circ} 07^{\prime} \mathrm{N} 78^{\circ} 38^{\circ} \mathrm{O}, 1250 \mathrm{~m}\right), 2$ o., 17.VIII.1991, P. Ward col. (MIZA).

\section{Gnamptogenys regularis (Mayr, 1870)}

Diagnosis. Cara en declive del propodeo abruptamente separada de la cara dorsal, superior lateralmente con pequeños lóbulos angulados; diente metacoxal apicalmente redondeado y no triangular; cuerpo café oscuro, patas testáceas.

Material examinado. COLOMBIA, Magdalena: $4 \mathrm{~km} \mathrm{~N}$ San Pedro $(550 \mathrm{~m}), 1$ o. (MIZA); Valle: Medio Calima (Campamento Campo Alegre, 1000 m), 1 o., XI.1993, R. Aldana col. (IAVH). ECUADOR, Pichincha: Río Palenque (Centro Científico, Santo Domingo de los Colorados), 1 o. (MIZA).

\section{Gnamptogenys relicta (Mann, 1916)}

Diagnosis. Mandíbulas, vértice y cara anterior pospeciolar lisos y brillantes, occipucio algunas veces con costillas transversas muy tenues; últimos tres segmentos antenales forman vagamente un mazo; cara en declive del propodeo con costillaje transverso, espiráculos propodeales elevados; sutura pronotal presente aunque fina; sutura metanotal bien impresa, rompiendo la escultura; sin lóbulos anterolaterales sobre la cara en declive del propodeo; espina metacoxal presente.

Material examinado. COLOMBIA, Amazonas: $7 \mathrm{~km} \mathrm{~N}$ Leticia, 1 o., 10-25.II.1972, S. \& J. Peck col., B-230 (MIZA).

\section{Gnamptogenys stellae Lattke, 1995}

Diagnosis. Cabeza y cuerpo con finas estrías longitudinales, apertura del espiráculo metatóracico montado sobre protuberancia cónica, ojos compuestos reducidos.

Material examinado. COLOMBIA, Valle: Puerto Merizalde, 1 o., XII.1988, M. Baena col., MQC-04 (IAVH).

Comentarios. Hasta ahora se conocía solamente del holotipo de Costa Rica.

\section{Gnamptogenys striatula Mayr, 1883}

Diagnosis. Costillaje pronotal semicircular; costillaje longitudinal dorsal y posteriormente sobre el mesosoma, ligeramente divergiendo caudalmente; mesometanoto algunas veces con costillas semicirculares; diente metacoxal triangular; cuerpo café oscuro.

Material examinado. COLOMBIA, Amazonas: P.N.N Amacayacu (Boca Caño Mata-Mata), 1 o., 21.IX.1988, F Fernández col. (MIZA); Antioquia: Mpio Frontino (P.N.N Orquídeas, Norte Cabaña Venados, 900m), 1 o., 6.IV.1996, E. Palacio col., EPIC-PON 00996 (IAVH); Mpio S. Luis (Río Claro, $\left.5^{\circ} 57^{\prime} \mathrm{N}, 74^{\circ} 51^{\prime} \mathrm{W}, 430 \mathrm{~m}\right), 1$ o., 23.I.1999, D. Campos col. (IAVH); Providencia (Estación Biológica), 1977, 10 o., 2 r., 2 m., C. Kugler col. (MIZA); Caldas: Samaná (La Miel, $500 \mathrm{~m}$ ), 1 o., 18.II.1994, A. Ordóñez col. (IAVH); Cundinamarca: Qda Chirihara (1400 m), 4 o., 25.VII.1976, W.L. Brown col. (MIZA); Fusagasugá, 1 o., IX.1991, Lucy col. (MIZA); La Vega, 1 o., IX.1991, J. Berrío col. (MIZA); Chocó: Ríosucio (La Balsa, $7^{\circ} 26^{\prime} \mathrm{N} 77^{\circ} 20^{\prime} \mathrm{O}, 70 \mathrm{~m}$ ), 1 o., F. Fernández col. (IAVH); Guaviare: R. Nukak Maku Moyano (Caño Cocuy, $2^{\circ} 11^{\prime} \mathrm{N} 7^{\circ} 11^{\prime} \mathrm{O}$, 1 o., II.1996, F. Escobar col. (IAVH); Meta: P.N.N. La Macarena (La Curría, 580 m), 1 o., VI.1990, E. Palacio col. (EPIC); (Caño La Curía, $570 \mathrm{~m}$ ), 2 o. (IAVH); Mesetas (La Uribe, $720 \mathrm{~m}$ ), 1 o., 30.IX.1989, F. Fernández col. (MIZA); Río Guayabero (Isla Angostura, 270 m), 14 o., 2 r., 3 m., 13.I.1977, C. Kugler col. (MIZA); Nariño: Quianga, 1 o., M. Baena col. (IAVH); Valle: Bajo Anchicayá, 1 o., III.1990, M. Baena col. (IAVH); San Cipriano, 1 o., VIII.1989, M. Baena col. (UDVC); R.F. Escalerete $(180 \mathrm{~m}), 1$ o., VIII.1995, R. Aldana col. (UDVC); El Vitícola, $180 \mathrm{~m}$ ), 1 o., 17.IX.1994, I. Ambrecht col. (UDVC); Bajo Anchicayá $(270 \mathrm{~m}), 1$ o., TH-BA-01, ex-contenido estomacal de Tamandua mexicana (IAVH); Montebello (cerca Cali, $1200 \mathrm{~m}$ ), 14.IX.1975, 2 o., 2 r., J. Lattke col. (MIZA); Cali (1000 m), 13.IX.1975, 17 o., 1 r., J. Lattke col. (MIZA); Bajo Calima (Buenaventura, 50 m), 1 o., 5.V.1996, R. Bertele col., BC-RB-01 (UDVC); Sevilla, 1 o., 9.I.1976, W. MacKay col. (MIZA); Bosque de Yotocó (1575 m), 1 o., 23.VI.1989, W. MacKay col. (MIZA); Quindio: alrededores Armenia, 1 o., 1.V.1993, J. Villegas col. (MIZA). ECUADOR, Napo: Limoncocha $(250 \mathrm{~m}), 3$ o., S. \& J. Peck col., B-348, 18.VI.1976 (MIZA); Pichincha: Sushijindi (Sucuá, 1500 m), 1 o. (MIZA).

\section{Gnamptogenys strigata (Norton, 1871)}

Diagnosis. Con costillaje tosco; superficie pospeciolar anterior plana y con 3 a 4 costas transversas; pelos largos, semi-decumbentes y semi-erectos abundantes sobre el cuerpo.

Material examinado. COLOMBIA, Antioquia: Urrao (P.N.N. Orquídeas, 1430 m), 1 o., 4.IV.1996, E. Palacio col., EPICO4 (EPIC); Caldas: Aguadas (Los Naranjos, $2220 \mathrm{~m}$ ), 1 o., XI.1994, C. Sarmiento col. (FFIC); Caquetá: P.N.N. Los Picachos (1250 m), 1 o., 5.XII.1997, F. Escobar col. (IAVH); Cundinamarca: Tena (Vereda Patio Bonito, Laguna de Pedro Palo, $2100 \mathrm{~m}$ ), 1 o., 26.IX.1994, D. Campos col. (IAVH); Nariño: Barbacoas (Tajadas, $1000 \mathrm{~m}$ ), 1 o., 1.V.1994, F. Escobar col. (IAVH); R.N. La Planada (Parcela Olga, $1^{\circ} 15^{\prime} \mathrm{N}$ 
$\left.78^{\circ} 15^{\prime} \mathrm{O}, 1850 \mathrm{~m}\right), 1$ r., 16-VII-2.VIII.2000, G. Oliva col. (IAVH); Norte de Santander: P.N.N. Tamá (Alto de Herrera, Vereda El Diamante, $7^{\circ} 07^{\prime} \mathrm{N} 72^{\circ} 13^{\prime} \mathrm{O}, 1450$ m), 1 o., IX.1999, E. González col. (IAVH); Risaralda: P.N.N. Ucumarí (La Suiza, 1750 m), 1 o., Usma \& R. Aldana col. (IAVH); Valle: El Ensueño (1620 m), 1 o., 8.XII.1993, M.L. Baena col., en tronco (IAVH); Pance $(1700 \mathrm{~m}), 1$ o. (UDVC); Reserva Yotoco, 2 o., F. Fernández col. (FFIC, MIZA); Bosque Yotocó (1575 m), 17 o., 23.VI.1989, W. MacKay col. (MIZA); Bosque de Yotoco, 1 o., 6.I.1984, W. MacKay col. (MIZA); Pance (1400 m), 1 o., II.1971 (MIZA); $(1700 \mathrm{~m}), 3$ o.,16.VI.71, W. Brown col. (MIZA); Calima Medio, 2 o., III.1988, F. Fernández col. (MIZA); Bajo Calima, 2 o., VII.1988, W. MacKay col. (MIZA); P.N.N. Los Farallones (Pico de Aguila, $1600 \mathrm{~m}$ ), 1 o., 16.IV.1992, M. Gamboa col. (MIZA).

\section{Gnamptogenys sulcata (Smith, 1858)}

Diagnosis. Mandíbulas semi-triangulares; costillaje mesosomal dorsal convergente caudalmente y transverso sobre la cara en declive del propodeo; diente metacoxal ausente, como mucho un tubérculo pequeño o lóbulo corto presente; color variable.

Material examinado. COLOMBIA, Antioquia: Mpio S. Luis (Río Claro, 557’N 7451'O, 430 m), 1 o., 20.I.1998, D. Campos col. (IAVH); Caquetá: Pto. Solano (P.N.N. Chiribiquete, $\left.0^{\circ} 12^{\prime} \mathrm{N} 72^{\circ} 25^{\prime} \mathrm{W}, 300 \mathrm{~m}\right), 1$ o., 19-22.II.2001, M. Ospina \& E. González col. (IAVH); Casanare: [sin más datos] 1 o. (MIZA); Cauca: Isla Gorgona (Poblado), 1 o., V.1990, M. Baena col. (UDVC); Chocó: Ríosucio (La Balsa, 70 m), 3 o., F. Fernández col. (1, FFIC, 2 IAVH); Magdalena: $12 \mathrm{~km}$ ESE Minca (780 m), 1 o. (MIZA); P.N.N. Tayrona (Pueblito, $300 \mathrm{~m}), 1$ o., F. Fernández col. (FFIC); (Pueblito, límite sur, $300 \mathrm{~m}$ ), 1 o., 26.I.1978, C. Kugler col. (MIZA); Meta: Vista Hermosa (S Villavicencio, Caño El Buque, 480 m), 1 o., 25.XII.1975, W. \& E. MacKay col. (IAVH); Nariño: La Guayacana, 1 o. (MIZA); Barbacoas (Tajadas, 1000 m), 1 o., 1.VII.1994, F. Escobar col., $\mathrm{n}^{\circ} .431$ (IAVH); Sucre: Colosó (Estación Primatológica), 1 o. (FFIC); Valle: $64 \mathrm{~km} \mathrm{E}$ Buenaventura $(570 \mathrm{~m}), 1$ o. (MIZA); Calima Medio (550 m), 1 o., 1994, R. Aldana col. (UDVC); P.N.N. Farallones de Cali, $\left.3^{\circ} 26^{\prime} \mathrm{N} 76^{\circ} 48^{\prime} \mathrm{O}, 900 \mathrm{~m}\right), 5$ o., X.2000, S. Sarria, col. (IAVH). ECUADOR, Pichincha: Tinalandia (16 km SE Santo Domingo de los Colorados, 680 m), 5 o., VI.1975, S. \& J. Peck col. (MIZA); Río Palenque (Estación Universidad de Miami), 5 o., 30.VII.1987, G. Umphrey col. (MIZA); (Centro Científico Río Palenque), 1 o., 1981, S. Sandoval col. (MIZA).

\section{Gnamptogenys tortuolosa (Smith, 1858)}

Diagnosis. Mandíbulas semi-triangulares; lámina clipeal lateralmente angular y medianamente recta a ligeramente cóncava; propodeo con costillaje transverso; diente coxal pequeño, algunas veces como un lóbulo corto; tibias, fémures y escapos lisos y pulidos; cuerpo píceo, patas y antenas ferruginosas.

Material examinado. COLOMBIA, Amazonas: Buenos Aires (Trocha Salado), 1 o., 16.VII.1997, L. Mendoza col. (FFIC); P.N.N. Amacayacu (cerca Boca Caño Mata-Mata), 1 o., 1.VIII.1989, A. Medina col. (MIZA); (San Martín, 323'S $\left.70^{\circ} 6^{\prime} \mathrm{O}, 150 \mathrm{~m}\right), 1$ o., 29.XII.2000-6.I.2001, B. Amado col. (IAVH); Caquetá: Solano, P.N.N. Chiribiquete (Río Sararamano, B. Verde Militar, $0^{\circ} 10^{\prime} 48^{\prime \prime} \mathrm{N} 72^{\circ} 37^{\prime} 24$ 'O, 300 m),1 o., 4.VII.2000, E. González col. (IAVH); Chocó: Riosucio (La Balsa, 70 m), 1 o., X.1994, L. Mendoza col. (FFIC); Guaviare: R.N. Nukak Maku (Caño Cucuy, $2^{\circ} 11^{\prime} \mathrm{N} 71^{\circ} 11^{\prime} \mathrm{O}$, 200 m), 1 o., II.1996, F. Fernández col. (IAVH); Meta:
Villavicencio (Bosque Bavaria, 545 m), 1 o., 29.XI.1996, C. Estrada col. (IAVH); (8 km W Villavicencio), 1 o. (IAVH); Mesetas (La Uribe, 720 m), 1 o., 30.XI.1987, F. Fernández col. (MIZA); (San Isidro, 340 m), 4 o., 11.II.1988, F. Fernández col. (MIZA); (San Isidro, vega Río Duda), 3 o., 19.II.1988, W. Cubillos col. (IAVH); Vista Hermosa (Refugio El Charro), 1 o., 10.IX.1987, F. Fernández col. (MIZA). ECUADOR, Pichincha: Sucua, 1 o. (MIZA).

\section{Gnamptogenys triangularis (Mayr, 1887)}

Diagnosis. Sutura promesonotal débilmente impresa; dorso del nodo con costillas transversas; proceso subpeciolar semi-cuadrado; primer esterno gástrico con costillaje transverso; diente metacoxal largo y delgado; cuerpo píceo.

Material examinado. COLOMBIA, Antioquia: Mpio San Luis (Río Claro, 557’’ 7451'O, 430 m), 1 o., 23.I.1998, D. Campos col. (IAVH); Chocó: Ríosucio (La Balsa, $70 \mathrm{~m}$ ), 6 o., F. Fernández col. (2 FFIC, 2 IAVH, 2 UNCB); Magdalena: $3 \mathrm{~km}$ SE Minca $(1050 \mathrm{~m}), 1$ o., P. Ward col. (PWIC); P.N.N. Tayrona (Pueblito, $300 \mathrm{~m}$ ), 1 o., 11.IX.1976, C. Kugler col. (MIZA). ECUADOR, Pichincha: $47 \mathrm{~km}$ S Santo Domingo de los Colorados (Estación Río Palenque), 3 o., 23.V.1975, S. \& J Peck col. (MIZA).

Agradecimientos. A Martha L. Baena, Rosa Aldana, Inge Armbrecht, Patricia Chacón (UDVC), Andrea Molano, Elvia L. Gonzalez (IAVH), Saulo Usma, Javier Bustos, Francisco Castaño (in memorian), Carlos Sarmiento, Luisa F. Mendoza, Catalina Estrada, Federico Escobar (IAVH), Miguel Rodríguez M., Giovanni Ulloa, Germán Domingo Amat (UNCB), William Cubillos por suministranos material o darnos acceso a sus colecciones. A W. L. Brown Jr (in memorian), C. R. F. Brandão, C. Kugler, W. P. MacKay y P. S. Ward por sus valiosos aportes de material y consejos.

\section{REFERENCIAS BIBLIOGRAFICAS}

Brandão, C. R. F. \& LattKe, J. E. 1990. Description of a new Ecuadorian Gnamptogenys species with a discussion on the satus of the Alfaria group. Journal of the New York Entomological Society, New York, 1998:489-494.

Brown, W. L., JR. 1958. Contributions toward a reclassification of the Formicidae. II. Tribe Ectatommini. Bulletin of the Museum of Comparative Zoology, Cambridge, 118(5): 175-362.

Fernández, F.; Palacio, E. E. \& Mackay, W. P. 1996. Introducción al estudio de las hormigas de Colombia. In: Andrade, G.; Аmat, G. \& Fernández, F. eds. Insectos de Colombia: estudios escogidos. Santafé de Bogotá, Academia Colombiana de Ciencias. p.349-412.

LattKe, J. E. 1990. Revisión del género Gnamptogenys Mayr para Venezuela. Acta Terramaris, Maracay, 2:1-47.

1991. Revision of the minuta group of the genus Gnamptogenys. Deutsche Entomologische Zeitschrift, Berlin, 39:129-139.

1994. Phylogenetic relationships and classification of the Ectatommini. Entomologica Scandinavica, Copenhagen, 25:105-119.

1995. Revision of the ant genus Gnamptogenys in the New World (Hymenoptera: Formicidae). Journal of Hymenoptera Research, Washington, 4:137-193.

2003. A taxonomic revision and phylogenetic analysis of the ant genus Gnamptogenys Roger in Southeast Asia and Australasia (Hymenoptera: Formicidae). University of California Publications in Entomology, Los Angeles, 122:1-281.

Recebido em abril de 2003. Aceito em julho de 2004. ISSN 0073-4721 